\title{
A Network Learning Approach for Asset Management in Water Distribution Infrastructure
}

\section{Zoe J. Y. Zhu and Edward A. McBean}

The combination of factors including the aging of water distribution infrastructure, growth in water demands, and limited operating budgets have created interest in more robust and rigorous methodologies to prioritize rehabilitation and renewal decisions for water distribution infrastructure. One such procedure, probabilistic network modeling, can be used to investigate issues of water infrastructure failure and to develop inclusive and dynamic analyses. Decomposable Markov networks (DMNs) and machine learning techniques in the domain of water distribution systems are developed herein. A framework is described that assesses causality or correlation between pipe breaks and relevant factors based on data from the Greater Toronto Area (GTA). The role of factors such as cement mortar lining (CML), soil type, pipe material and dimension are employed. The framework can be used to assist decision-making by estimating probabilities of future pipe breakage and identifying rehabilitation options to decrease breakage probabilities. The DMN-based machine learning approach provides an excellent way of combining engineering knowledge and available data in a robust and formal statistical manner.

Zhu, Z.J. and E. McBean. 2009. "A Network Learning Approach for Asset Management in Water Distribution Infrastructure." Journal of Water Management Modeling R235-24. doi:

10.14796/JWMM.R235-24.

(C) CHI 2009 www.chijournal.org ISSN: 2292-6062 (Formerly in Conceptual Modeling of Urban Water Systems. ISBN: 978-0-9808853-2-3) 


\subsection{Introduction}

Water distribution infrastructure systems in many countries are aging virtually with calendar time. For example, Schuster and McBean (2007) found that infrastructure in the Greater Toronto Area (GTA) has been aging at a rate of just over $7 \mathrm{y} /$ decade since 1960. Further complicating the challenges of maintaining water distribution systems are substantial increases in population and the tightening of municipal budgets.

It is estimated that nearly $50 \%$ of GTA's pipe network was laid over 50 years ago. Pipe materials employed include cast iron, ductile iron (DI) and asbestos cement pipes. All these materials suffer from degradation over time due to operational measures, environmental conditions and general wear and tear and result in increased leakage in the network. During the winter of 1995, Scarborough, a suburb of Toronto, experienced more than 160 breaks, (McBean, 2006).

Hence, questions of asset management involving repair and replacement must incorporate deterioration and failure of water pipes through appropriate predictive models of infrastructure components. Understanding the role of contributing factors which influence performance and the life of water distribution infrastructure has been ongoing, in part because the investments in infrastructure are large and pipe breakages are frequent. Specifically, for three municipalities, namely East York \& York, Etobicoke, and Scarborough, all in the Toronto area, data range from 1901 to 2002. The pipebreak frequency is increasing per $100 \mathrm{~m}$ length from 0.27 to 0.35 for the first break, 0.16 to 0.26 for the second break, and 0.11 to 0.19 for the third break as shown in Table 24.1.

Table 24.1 Pipe break frequency.

\begin{tabular}{cccccccc}
\hline $\begin{array}{c}\text { Time of } \\
\text { Placement }\end{array}$ & $\begin{array}{c}\text { Length } \\
(\mathrm{m})\end{array}$ & $\begin{array}{c}\text { First } \\
\text { Break } \\
\text { (times) }\end{array}$ & $\begin{array}{c}\text { 1st Break } \\
\begin{array}{c}\text { Frequency } \\
\text { (per 100m) }\end{array}\end{array}$ & $\begin{array}{c}\text { Second } \\
\text { Break } \\
\text { (times) }\end{array}$ & $\begin{array}{c}\text { 2nd Break } \\
\text { Frequency } \\
\text { (per 100m) }\end{array}$ & $\begin{array}{c}\text { Third } \\
\text { Break } \\
\text { (times) }\end{array}$ & $\begin{array}{c}\text { 3rd Break } \\
\text { Frequency } \\
\text { (per 100m) }\end{array}$ \\
\hline $1952-2002$ & 665923.7 & 5181 & 0.35 & 3968 & 0.26 & 2799 & 0.19 \\
$1901-1951$ & 832550.1 & 2283 & 0.27 & 1359 & 0.16 & 915 & 0.11 \\
\hline
\end{tabular}

An integral component of effective asset management involves gaining an understanding of the causative features of pipe breaks and impacts of preventive measures such as pipe relining and cathodic protection. Water pipe-leakage and pipe-breaks result not only in disruption of service, 
exposure risks of water consumers, and also potentially significant loss of water. As an indication, water loss in distribution systems is estimated on average as approximately $20 \%$ of the water produced by municipalities in Canada. This water is lost or "unaccounted for", mostly due to distribution system leakage (Environment Canada, 2004). In the US, unaccounted for water is reported to range from 15 to $25 \%$, of which about $60-75 \%$ is recoverable leakage (Vickers, 1999). Reported levels of losses are generally underestimated and increase with the age of distribution systems. Losses in Montreal's distribution system are reported to be at least $40 \%$ (Beauvais, 2005).

Pipe leakage and breaks also create public health exposure risks as a result of contaminants which may enter the break if water pressures in portions of the distribution system are lost. Pathogens, through pipe breaks, bypass treatment processes (Geldreich, 1988). Chlorine residual is maintained within the distribution system to limit exposure risks but taste and odour problems and the potential for chlorine to react with dissolved organic carbon to produce disinfection by-products (e.g. trihalomethanes), place restrictions on the amounts of disinfectant which are added to water in distribution systems (Zhu et al., 2005).

To improve asset management decisions, including improvement in decision-making related to rehabilitation and replacement of pipes, an understanding of the causative factors for pipe failure and the influences of contributing factors can be obtained by a modeling framework. Due to the complex inter-relationships which exist, the ideal approach is to use a model developed from historical data; one such procedure, described herein, is based upon probabilistic networks and more specifically, Decomposable Markov Networks (DMNs).

This chapter describes how the DMN systematically evaluates pipe breaks within the water distribution system and establishes dependency on factors which influence pipe breakage frequency. A DMN-based machine learning method is used herein to construct the model from historical data on pipe break frequency. The procedure then uses the model to predict when a pipe of a given condition is likely to break and when it is most cost-efficient to replace the pipe.

\subsection{Historical Approaches and Background}

Failure analyses have been used to quantify the historical incidence of pipe failures in terms of failure rates and inter-failure times to examine reliability and remaining life by a number of researchers including Shamir and Howard 
(1979), O’Day et al. (1980), Clark and Goodrich (1988), Anderson et al. (1987), and Gustafson and Clancy (1999). Research has included, for example, that a pipe that is scheduled for relining due to inadequate hydraulic capacity may be better replaced if its breakage frequency is expected to rise significantly in the future (Engelhardt et al., 2000). The challenge is, in part, to determine the most appropriate plan in terms of replacement of a pipe, versus rehabilitation, versus repair as breaks occur.

All of these models are useful but are limited, for a variety of reasons, because of the use of integer variables, reliance upon particular probability distributions, and the use of simple conditional dependence. As an alternative, a different approach is to rely upon network models. Decomposable Markov Network models build upon the extensive capabilities of probabilistic network modeling theory and provide an opportunity to employ very flexible procedures.

Decomposable Markov Networks (DMNs), Bayesian networks (BNs) and machine learning are powerful tools developed in the field of artificial intelligence for modeling and decision-making in uncertain problem domains. In groundwater remediation, BNs coupled with an expert knowledgebase with process models have been used to evaluate the potential of naturally occurring reductive dechlorination at sites contaminated with TCE. (Siber et al., 1999). Marcot (2001) combined expert knowledge with ecological data within a $\mathrm{BN}$ to model the causal relationships between planning decisions and impacts on at-risk wildlife species habitats. Stow et al. (2003) compared a BN approach with two deterministic models for predicting the effect of nitrogen loading on estuarine chlorophyll concentrations. Zhu and McBean (2004) employed DMN and machinelearning on censored data prediction and also developed water treatment decision procedures based on Bayesian decision networks (Zhu et al., 2007). Hence, DMNs can provide significant insight into asset management decision-making while avoiding some of the restrictions implicit in alternative procedures.

A $B N$ is a vector $(V, G, P)$ where $V$ is a set of domain variables, $G$ is a directed acyclic graph (DAG) whose nodes are labeled one to one by variables in $\mathrm{V}$ and whose links encode causal dependency between variables being connected. $\mathrm{P}$ is a set of conditional probability distributions, with one distribution $\mathrm{P}(\mathrm{x} \mid \operatorname{par}(\mathrm{x}))$ associated with each variable $\mathrm{x}$, where $\operatorname{par}(\mathrm{x})$ are the parents of $\mathrm{x}$ in $\mathrm{G} . \mathrm{P}(\mathrm{x} \mid \operatorname{par}(\mathrm{x}))$ quantifies the causal strength between $\mathrm{x}$ and $\operatorname{par}(\mathrm{x})$. Given a $\mathrm{BN}(\mathrm{V}, \mathrm{G}, \mathrm{P})$, the joint probability distribution over $\mathrm{V}$ is the following: 


$$
P(V)=\prod_{v \varepsilon V} P(v \mid \operatorname{par}(v))
$$

Figures 24.1 and 24.2 show an example $\mathrm{BN}$ for a drinking water system. Two potential causes of water contamination, bioterrorism and industrial wastes, as well as two effects of water contamination, dead fish and sickness of people, are depicted. $\mathrm{P}($ Bioterrorism=yes) encodes the likelihood of the threat. $\mathrm{P}($ WaterContamination $=\mathrm{y} \mid$ Bioterrorism=yes, Industrial wastes=yes) encodes the likelihood of water contamination given that both causes are active.

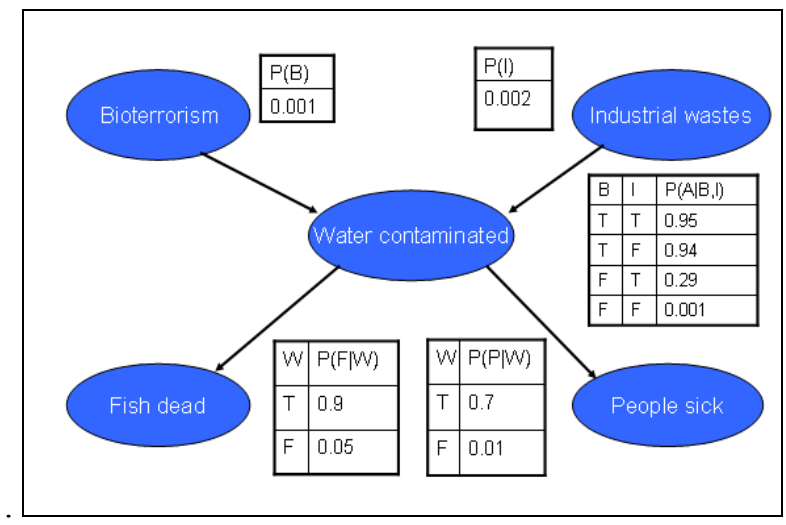

Figure 24.1 Example of Bayesian networks.

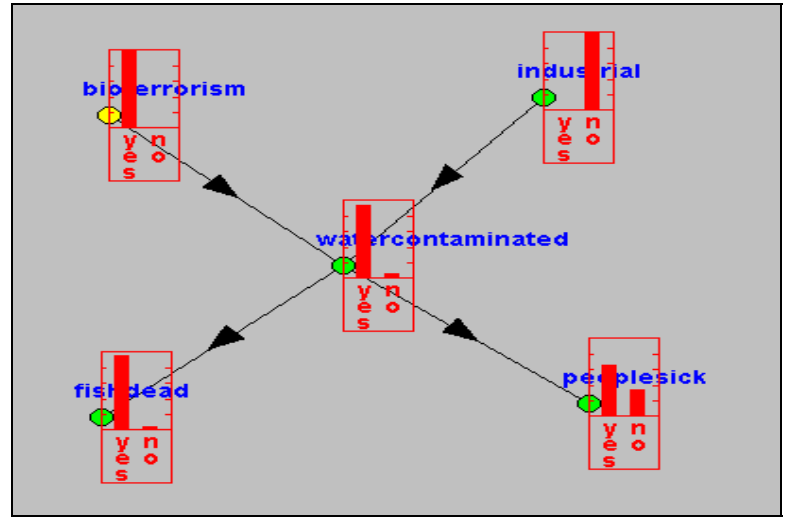

Figure 24.2 Probability distribution if bioterrorism = yes. 
Figure 24.2 shows the probability distribution if Bioterrorism =yes. If we know that dead fish= yes, we also can derive the probability distribution of other variables such as industrial waste.

A DMN is similar to a BN but is an undirected graph. The graph (see Figure 24.3) consists of Clique + Separators and it is called a clique graph. The intersection of any two cliques is contained in each clique on the path between them. The joint probability distribution (JPD) is the product of clique distributions divided by the product of separator distributions.

Consider a simple example as shown in Figure 24.3. Watermain breakage may cause the drinking water distribution system to be contaminated. Absence of backflow devices may cause backflow of water, which in turn may cause contamination of the drinking water distribution system. Human sickness may occur due to such contamination.

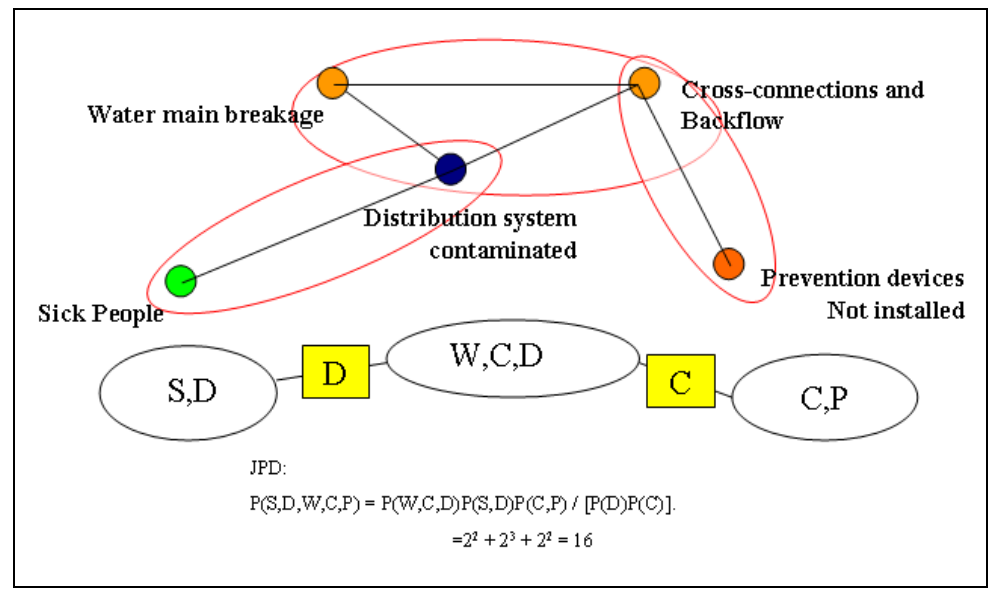

Figure 24.3 An example of DMN.

When a DMN or BN model is developed, the qualitative structure of the domain (the graph which encodes the dependence and independence relations among variables) is specified and the influences (the probability parameters) are quantified. Typically, the most difficult and time-consuming part of the task in building a DMN or BN model is specifying the structure. Probabilities can be derived from various sources: (i) they can be obtained by interviewing domain experts to elicit their subjective probabilities; (ii) they can be gathered from published statistical studies or can be derived analytically from well-founded theories, for example, how 'genes' transmit 
from 'parents' to 'children'; and (iii) they can be estimated from raw data. (Zhu and McBean, 2007). Since for a given number of variables, there are many more BN models than DMN models, learning DMNs are computationally more efficient. Learning DMNs from raw data are adopted in this research.

\subsection{Methods}

Application of DMNs to water distribution system breakage frequency prediction can be divided into two stages: a 'learning' stage and an 'inference' stage. In the learning stage, a DMN model is developed over a set of variables from 'training' data. In the 'training' data stage, every variable has a value. To 'learn' the structure from training data, both a scoring metric and a search procedure are employed. The scoring metric evaluates each structure and selects the resulting structure with the highest score or dependence. The search procedure generates alternative graphical structures which encode alternative sets of dependence and independence relationships.

The data for each variable in the training dataset is discretized into 'bins' and the correlation structure between the variables is 'learned' and encoded as a DMN, specifying the Joint Probability Distribution (JPD) over the domain through chordal graphs and the local probability distribution associated with each clique (Zhu and McBean, 2004).

WEBWEAVR-IIII Toolkit (after Xiang 1997, and Haddaway 1999) can be used for construction of the DMNs, and subsequently, for inference, with these models. Algorithms implemented in WEBWEAVR-IIII Toolkit are based on rigorous mathematical analyses and verification. In the learning stage, a total of 5764 data records from pipe break frequency from E. York, Etobicoke, Scarborough and York regions in Ontario. The data involve 1684 Cast Iron (CI) pipes, 3020 Ductile Iron (DI) pipes, 258 Asbestos Cement (AC) pipes, and 700 Polyvinyl Chloride (PVC) pipes and 98. Concrete (Conc.) piples. The resultant learned DMN is illustrated in Figure 24.4 showing the linkage between alternative structured variables, namely length, diameter and pipe material. Remedial measures, namely $\mathrm{CP}$ and CML refer to cathodic protection and cement mortar lining applied to the pipe. Soil refers to the type of soil where the pipe is embedded. Agebrk1 refers to the year-range when the first breakage of the pipe occurs.

In the inference stage, investigations can be completed to predict the timing of future pipe breakage, for given conditions of soil type, age, pipe material. For example, to predict timing of future pipe breakage, the soil 
condition or material of the pipe may be entered into the corresponding cliques of the DMN. After probabilistic reasoning in the DMN, the posterior probability distribution of each unknown variable, including the time of future breakage can be retrieved from the DMN. The second task of the inference stage involves exploring the causal or correlation relationship between a target variable and other variables and identifying constituents that lead to increases or decreases in the likelihood of breakage. For example, the pipe material type can be entered into the DMN as Cast Iron (CI) in one session and as Ductile Iron (DI) in a second session.

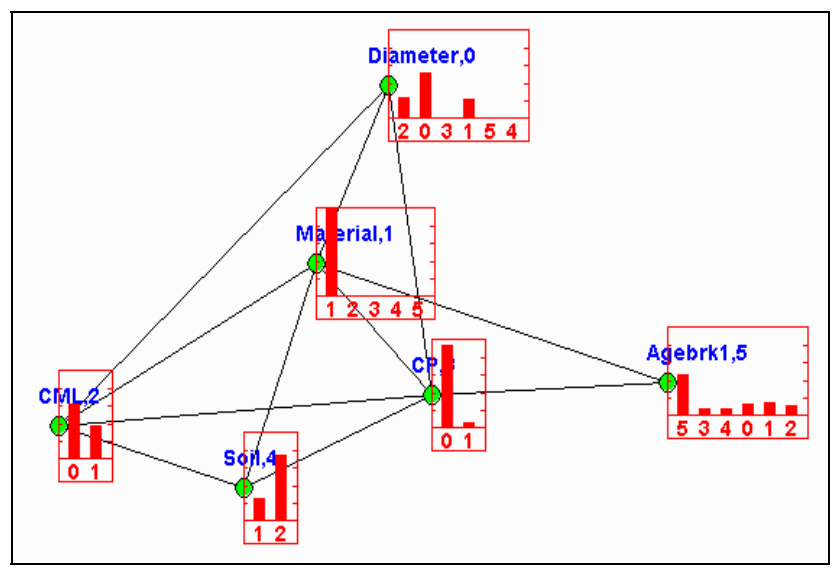

Figure 24.4 DMN with alternative structured variables.

The probability distribution of future breakages will be estimated. Because DI is a better material compared with CI, the posterior probability distribution of breakage obtained from the second assessment will show lower probabilities than the CI. As a DMN can support uncertain inference of any target variables, we can also assume the future breakage and analyze how states of independent variables, such as material and soil conditions, can influence pipe breakage probabilities.

\subsection{Effect of Pipe Material on First Pipe Break}

The DMN results indicate that the first pipe break directly depends on the pipe material as indicated by the direct link between Agebrk1 and Material (Figure 24.4). To quantify the dependence, the different values for Material (representing the different material types) are entered into the DMN and the 
updated probability distribution of Agrbrk1 is computed. The numerical distributions for CI, DI and AC., etc. are summarized in Figure 24.5.

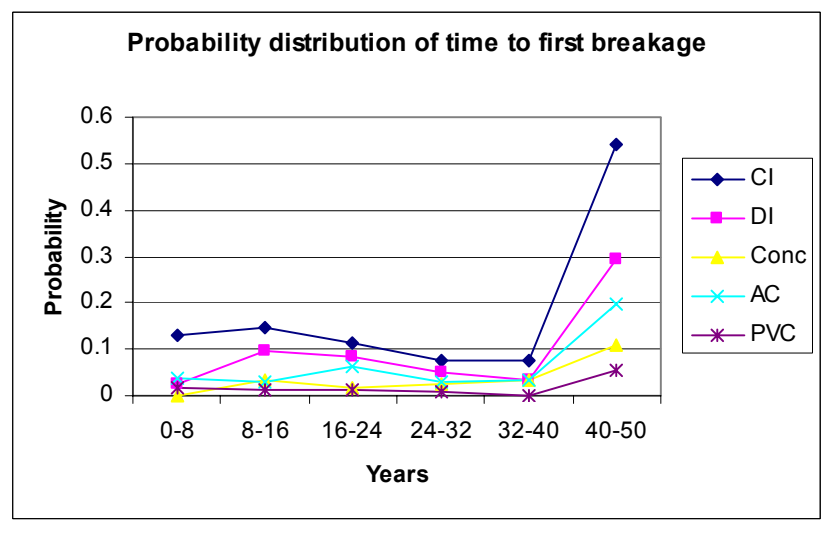

Figure 24.5 Impact of pipe material type to first pipe break.

As apparent from Figure 24.5, within 8 y of placement in the ground, 13\% of CI pipes, $3 \%$ of DI pipes, $0 \%$ of Conc., $4 \%$ of $\mathrm{AC}$ pipes and $1.7 \%$ of $\mathrm{PVC}$ will break, indicating that DI and AC pipes take longer to break than CI pipes. These findings are consistent with NRC studies: Cast iron pipes yielded the highest failure rate compared to other materials, such as DI, AC and PVC; and PVC pipe exhibits the lowest failure rate of pipe installed today.

Comparing the two iron-based pipes, CI pipe is relatively brittle and easily broken or cracked by applied pressures especially when external bending moments are applied by soil movement. Additionally, CI pipe is the most susceptible to breaks due to freezing. DI pipe is both stronger and less brittle than cast-iron pipe. AC pipes, while no longer installed, posted the second lowest failure rate. Their yearly break rate was 5.8 breaks per $100 \mathrm{~km}$ of AC pipe in service. Their lower failure rate may be due to their inherent noncorroding nature. Using NRC failure data, PVC pipes offer the best value requiring an average of $C \$ 1,750$ per $100 \mathrm{~km}$ in repair costs - about fourteen times less than is now the case with DI pipes.(NRC, A-7019.1).

\subsection{Effect of CP to First Pipe Break}

Iron-based pipes have inherently high structural strengths but they are prone to corrosion processes. Corrosion is an electrochemical reaction between 
two materials in contact with each other that have a difference in potential, allow the transfer of electrons from one to the other, and result in the loss of parent material. Corrosion has many causes, including dissimilar soils adjacent to the pipe with unequal moisture, differing levels of $\mathrm{pH}$, soil bacteria and surface scratches that lead to stress points and strain patterns at the microscopic level which can be the initiating places for corrosion. The result of internal and external corrosion will increase pipe breakage frequencies as well as impacts on water quality and the hydraulic performance of the pipe system. Furthermore, corrosion is almost always a necessary precursor to other types of failure modes. AC pipes may also undergo a chemical reaction internally and externally. If the carbon dioxide/calcium carbonate content of the pipe water is unbalanced, the calcium carbonate content of the cement will be leached out, with an accompanying reduction in the structural strength of the pipes.

$\mathrm{CP}$ is a method to reduce corrosion by minimizing the difference in potential between anode and cathode by applying an electric current to the pipes. DMN analyses demonstrate that $\mathrm{CP}$ reduces the probability of pipe breaks (Table 24.2). Specifically, during the first $8 \mathrm{y}$ the probability of a first break decreased from $13 \%(\mathrm{CP}=0)$ to $11 \%(\mathrm{CP}=1)$ for $\mathrm{CI}$.

Table 24.2 Cathodic Protection impact on the first pipe break.

Case 1: $\mathrm{CP}=0$

\begin{tabular}{lllll}
\hline $0-8$ & $8-16$ & $16-24$ & $24-32$ & $32-40$ \\
\hline 0.13 & 0.14 & 0.11 & 0.07 & 0.07 \\
\hline
\end{tabular}

Case 2: $\mathrm{CP}=1$

\begin{tabular}{lllll}
\hline $0-8$ & $8-16$ & $16-24$ & $24-32$ & $32-40$ \\
\hline 0.11 & 0.21 & 0.12 & 0.10 & 0.05 \\
\hline
\end{tabular}

\subsection{The Causal Relationship between First and Second Pipe Breaks}

For the available data, 1292 pipes out of 1977 pipes (approx. 65\%) experienced a second break. In this section, the relationship is explored between the first and the second breakages. The newly derived DMN, consisting of 1292 records, is shown in Figure 24.6. 


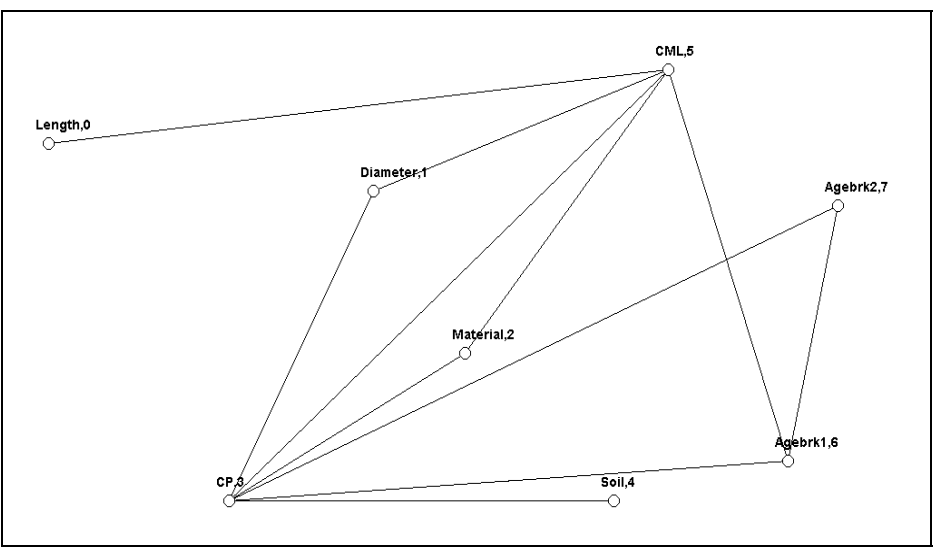

Figure 24.6 DMN network incorporating second breaks.

As expected, and as demonstrated in Figure 24.6, the second break is directly dependent on the first break. This dependency is further quantified in Tables 24.3 and 24.4. As shown in Table 24.3, given that the first pipe breakage occurred within $7 \mathrm{y}$ of pipe placement, $28 \%$ of pipes experience a second break within $7 \mathrm{y}$ of the first break for CI, and 38\% within $14 \mathrm{y}$. For $\mathrm{DI}$, the dependency is $25.1 \%$ within the next $7 \mathrm{y}$ and $41 \%$ within $14 \mathrm{y}$. (Table 24.4). From Table 24.3 for CI, it can also be seen that once the first breakage occurred, $66 \%$ of the pipes have the next breakage within the next $7 \mathrm{y}$, and $86 \%$ of the pipes have the next breakage within the next $14 \mathrm{y}$ (or less). The similar dependency can be seen from Table 24.4 for DI, at $66 \%$ and $84 \%$, respectively.

Table 24.3 Correlation between first breakage and second breakage for $\mathrm{Cl}$.

\begin{tabular}{ccccccc}
\hline Material CI & Agbrk2=1 & Agbrk2=2 & Agbrk2=3 & Agbrk2=4 & Agbrk2=5 & Agbrk2=65 \\
\hline Agbrk1=1 & 0.28 & 0.38 & 0.20 & 0.088 & 0.043 & 0.016 \\
Agbrk1=2 & 0 & 0.35 & 0.37 & 0.166 & 0.071 & 0.053 \\
Agbrk1=3 & 0 & 0 & 0.40 & 0.39 & 0.14 & 0.069 \\
(Agbrk1=4) & 0 & 0 & 0 & 0.48 & 0.38 & 0.14 \\
(Agbrk1=5) & 0 & 0 & 0 & 0 & 0.48 & 0.52 \\
(Agbrk1=6) & 0 & 0 & 0 & 0 & 0 & 1 \\
\hline
\end{tabular}

Legend: Bins of $7 \mathrm{y}$ in duration 
Table 24.4 Correlation between first breakage and second breakage for DI.

\begin{tabular}{ccccccc}
\hline $\begin{array}{c}\text { Material } \\
\text { DI }\end{array}$ & Agbrk2=1 & Agbrk2=2 & Agbrk2=3 & Agbrk2=4 & Agbrk2=5 & Agbrk2=6 \\
\hline Agbrk1=1 & 0.25 & 0.41 & 0.18 & 0.11 & 0.036 & 0.013 \\
Agbrk1=2 & 0.0 & 0.50 & 0.34 & 0.093 & 0.041 & 0.026 \\
Agbrk1=3 & 0.0 & 0.0 & 0.51 & 0.34 & 0.10 & 0.042 \\
Agbrk1=4 & 0.0 & 0.0 & 0.0 & 0.49 & 0.37 & 0.14 \\
Agbrk1=5 & 0.0 & 0.0 & 0.0 & 0.0 & 0.53 & 0.47 \\
Agbrk1=6 & 0.0 & 0.0 & 0.0 & 0.0 & 0.0 & 1 \\
\hline
\end{tabular}

Legend: bins of $7 \mathrm{y}$ in duration

These results also demonstrate that if a pipe has broken once, there is a high probability that it will break again, relatively soon thereafter.

\subsection{The Causal Relationship between Second and Third Pipe Breaks}

In the new DMN (Figure 24.6), the node for the third breakage is indirectly connected to the node for the first breakage through the node for the second breakage which is expected, since the third break can only occur following the second break. This means that the third breakage is conditionally independent of the first breakage given the second breakage, i.e. the occurrence and timing of a third break can be predicted solely from the second break. If the second pipe break happened within $7 \mathrm{y}$ of pipe placement, $53 \%$ of pipes experience a third break within $7 \mathrm{y}$ after the second break, and $30 \%$ within 14 y for CI (see Table 24.5). For DI, the dependency is $17 \%$ within $7 \mathrm{y}$ and $58 \%$ within $14 \mathrm{y}$, as shown in Table 24.6. Further, for CI, once the second break has occurred, at least $83 \%$ of the pipes experience another break within the next $7 \mathrm{y}$, and at least $99 \%$ of the pipes experience another break within the next $14 \mathrm{y}$. The similar dependency can be seen from Table 24.9 for DI, at $74.4 \%$ and $98.3 \%$, respectively. 


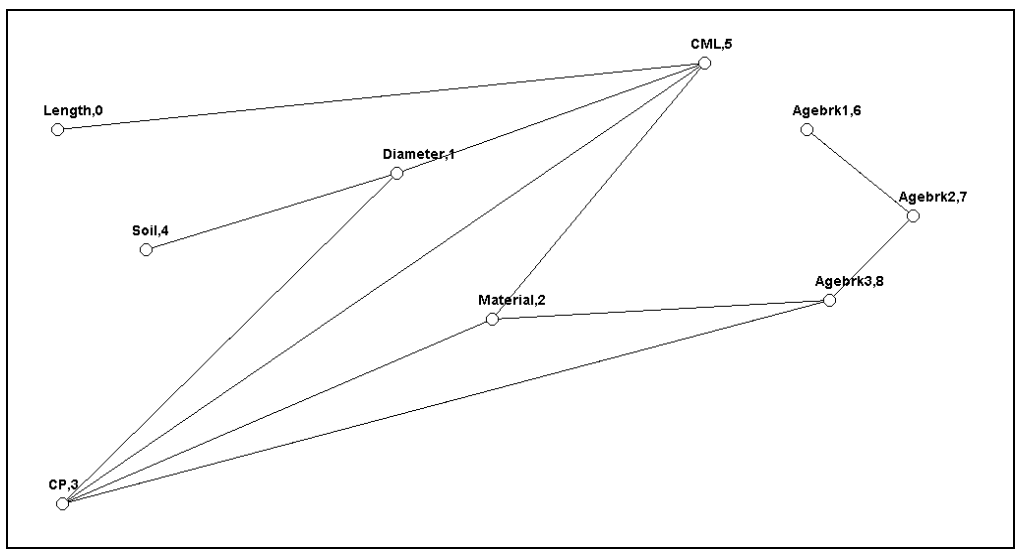

Figure 24. 6 DMN network with third breakage.

Table 24.5 Correlation between second breakage and third breakage for $\mathrm{Cl}$.

\begin{tabular}{ccccccc}
\hline $\begin{array}{c}\text { Material } \\
\text { CI }\end{array}$ & Agbrk2=1 & Agbrk2=2 & Agbrk2=3 & Agbrk4=3 & Agbrk33=4 & Agbrk2=5 \\
\hline Agbrk1=1 & 0.53 & 0.30 & 0.15 & 0.014 & 0.0 & 0.0 \\
Agbrk1=2 & 0.0 & 0.55 & 0.32 & 0.084 & 0.015 & 0.025 \\
Agbrk1=3 & 0.0 & 0.0 & 0.44 & 0.34 & 0.12 & 0.090 \\
Agbrk1=4 & 0.0 & 0.0 & 0.0 & 0.48 & 0.36 & 0.16 \\
Agbrk1=5 & 0.0 & 0.0 & 0.0 & 0.0 & 0.45 & 0.55 \\
Agbrk1=6 & 0.0 & 0.0 & 0.0 & 0.0 & 0.0 & 1.0 \\
\hline
\end{tabular}

Table 24.6 Correlation between second breakage and third breakage for DI.

\begin{tabular}{ccccccc}
\hline $\begin{array}{c}\text { Material } \\
\text { DI }\end{array}$ & Agbrk2=1 & Agbrk2=2 & Agbrk2=3 & Agbrk2=4 & Agbrk2=5 & Agbrk2=6 \\
\hline Agbrk1=1 & 0.17 & 0.58 & 0.24 & 0.015 & 0.0 & 0.0 \\
Agbrk1=2 & 0.0 & 0.63 & 0.30 & 0.056 & 0.0066 & 0.0051 \\
Agbrk1=3 & 0.0 & 0.0 & 0.58 & 0.32 & 0.077 & 0.027 \\
Agbrk1=4 & 0.0 & 0.0 & 0.0 & 0.62 & 0.31 & 0.065 \\
Agbrk1=5 & 0.0 & 0.0 & 0.0 & 0.0 & 0.64 & 0.36 \\
Agbrk1=6 & 0.0 & 0.0 & 0.0 & 0.0 & 0.0 & 1.0 \\
\hline
\end{tabular}




\subsection{Conclusion}

These analyses, based on the GTA pipe break database, demonstrate that:

1. CI pipes are prone to earlier failure than DI or other material types and that PVC has the best value.

2. $\mathrm{CP}$ is an effective remediation technique that prolongs the life of a pipe

3. Once a pipe breaks, it is very likely to break again

The DMN-based approach allowed the systematic structuring of "prior knowledge" from historical data through learning, in order to predict the likelihood of pipe failure at a particular future time interval and the useful pipe life. The data alone are inefficient for predicting future pipe conditions, so the objective of this work was to fit the data using scientifically plausible models.

Coupling DMN modeling with machine learning takes full advantage of two powerful tools. DMN models can be learned from raw data and the learned models can then be used to systematically analyze factors affecting pipe breaks within water distribution systems. The model can easily simulate various realistic or experimental scenarios, which makes it useful for planning, forecasting and maintaining the water distribution system. In this manner, we can fully utilize all relevant information in the most efficient manner while keeping within a formal statistical framework. Advantages include quantification of correlation and dependence relationships among relevant attributes and the broad applicability of the method. Uncertainty in the models could be reduced by use of additional data.

Thus, the approach provides increased accuracy in failure rate estimations as well as identifying the relative risk of environmental effects and stress factors that cause pipe failures, as demonstrated through the specific GTA example. Those findings should be a tremendous aid to water utilities seeking to enhance their operating efficiency.

\section{Acknowledgments}

The authors would like to thank the City of Toronto, particularly Robert Klimas and Abdul Syed, for provision of the pipebreak data. Funding was provided through the Joint Infrastructure Interdependency Research Program and Canada Research Chair funds. 


\section{References}

Afshar, Mohammad H. and Mariño, Miguel A. 'Application of an ant algorithm for layout optimization of tree networks', Engineering Optimization, 38:3, 353 - 369, 2006.

Al-Jasser,_A.O., Chlorine decay in drinking-water transmission and distribution systems: Pipe service age effect, Water Research, 41, 387 - 396, 2007.

Beauvais, A., Montréal accorde un contrat de $30 \mathrm{M} \$$ pour réparer les réseaux d'égouts et d'aqueduc, Le Journal de Montréal, January 21, 2005.

Charniak, E., "Bayesian networks without tears", AI Magazine, vol. 12 no. 4: 50-63, 1991.

Herskovits, E.H. and Cooper, G.F., "Kutato: an entropy-driven system for construction of probabilistic expert systems from database", In Proc. 6th Conf. on Uncertainty in Artificial Intelligence, Pages 54-62, Cambridge, 1990.

Clarke, R. M., Grayman, W. M., \& Deininger, R. A. Measuring and modeling chlorine propagation in water distribution systems. Journal of Water Resources Planning and Management Division ASCE, 120(6), 1994.

Engelhardt, M.O., et al, Rehabilitation strategies for water distribution networks: a literature review with a UK perspective, Urban Water, 2 153-170, 2000

Environment Canada, Threats to Water Availability in Canada, NWRI Scientific Assessment Report Series No. 3 and ACSD Science Assessment Series No. 1, National Water Research Institute, Burlington, p. 128. 2004,

Geldreich E. E., Coliform non-compliance in water supply distribution systems. In Water Quality: a realistic perspective, Michigan Section Amer. Water Works Assoc and Michigan Water Poll. Control Assoc. joint Projecl: Report, College of Engineering, U. Michigan, Ann, Arbor, MI, pp. 55-74, 1988

Heckman , D, Geiger, D. and Chickering D.M., "Learning Bayesian networks: the combination of knowledge and statistical data", Machine Learning 20:197-243, 1995.

Henrion, M., "Propagating uncertainty in Bayesian networks by probabilistic logic sampling", In J.F. Lemmer and L.N. Kanal, editors, Uncertainty in Artificial Intelligence 2, pages 149-163, Elsevier Science Publishers, 1988

Jensen, F.V., Lauritzen, S.L, and Olesen,K.G., "Bayesian updating in causal probabilistic networks by local computations", Computational Statistics Quarterly, (4):269-282, 1990.

Lam, W., and Bacchus, F. "Learning Bayesian belief networks: An approach based on the MDL principle”, Computational Intelligence 10(4): 269-293. 1994.

Lund, J.R., Israel, M., Optimization of transfers in urban water supply planning. J. Water Resour. Plann. Manage., Am. Soc. Civil Eng. 121 (1), 41-48, 1995.

McBean, E. A. Failure mechanisms and monitoring methodologies pertinent for detection of exposure risks in water distribution networks. Springer Verlag, Netherlands, volume, 2006.

Marcot, B. G., Using Bayesian belief networks to evaluate fish and wildlife population viability under land management alternatives from an environmental impact statement, Forest Ecology and Management, v 153 (1-3), n 1-3, pp. 29-42, 2001.

NRC, A-7019.1 Final Water Mains Break Data on Different Pipe Materials for 1992 and 1993 
Pearl, J., "Fusion, propagation, and structuring in belief networks, Artificial Intelligence",(29):241-288, 1986.

Peal, J., "Evidential reasoning using stochastic simulation of causal models". Artificial Intelligence, 32:247-257, 1987.

Peal, J., "Probabilistic reasoning in intelligent systems". 2nd. ed. San Francisco, Calif., Morgan Kaufmann, 1991.

Schuster, C., and McBean, E., "Impact of Cathodic Protection on Pipe Break Probabilities: A Toronto Case Study", Canadian Journal of Civil Engineering, forthcoming.

Siber, N. A., M. Pantazidou, and M. J. Small, Expert system methodology for evaluating reductive dechlorination at TCE sites, Environmental Science and Technology, v 33(17), pp. 3012-3020, 1999.

Stow, C. A., C. Roessler, M. E. Borsuk, J. D. Bowen, and K. H. Reckhow, Comparison of Estuarine water quality models for total maximum daily load development in Neuse River Estuary, Journal of Water Resources Planning and Management, v 129 (4), pp. 307-314, 2003.

Veldkamp, R.G., Hermann, T., Colandini, V., Terwel, L., Geldof, G.D., A decision network for urban water management. Wat. Sci. Technol. 36 (8-9), 111-115, 1997.

Vickers, A. The Future of Water Conservation: Challenges Ahead. Water Resources Update, No. 114, Universities Council on Water Resources, Carbondale, IL, Winter, available at: www.ucowr.siu.edu/updates/pdf/V114_A8.pdf, 1999.

Shamsi, U.M. 2006. "Modeling the Reliability of Water Distribution Systems." Journal of Water Management Modeling R225-12. doi: 10.14796/JWMM.R225-12.

Xiang,Y. Wong, S.K.M and Cercone, N., "A Microscopic study of minimum entropy search in learning decomposable Markov networks", Machine Learning, Vol.26, No.1, 65-92, 1997.

Xiang Y. and Lee, J., Learning Decomposable Markov Networks in Pseudo-Independent Domains with Local Evaluation. Machine Learning, Vol.65, No.1, 199-227, 2006.

Zhu Z. J. Y and McBean E. A., "Estimation of censored data water quality values using decomposable Markov networks", Journal of Environmental Informatics, 4 (2) 8-55, 2004.

Zhu Z. J. Y and McBean E. A, Bayesian Decision Network Analyses to Select between Water Treatment Processes, Journal of Environmental Engineering and Science, 6: 1-8 2007. 